\title{
The Influence of Device Geometry on Many-Body Effects in Quantum Point Contacts: Signatures of the 0.7 Anomaly, Exchange and Kondo
}

\author{
E.J. Koop • A.I. Lerescu • J. Liu • B.J. van Wees • \\ D. Reuter · A.D. Wieck $\cdot$ C.H. van der Wal
}

Received: 18 June 2007 / Accepted: 20 June 2007 / Published online: 29 August 2007

(C) Springer Science+Business Media, LLC 2007

\begin{abstract}
The conductance of a quantum point contact (QPC) shows several features that result from many-body electron interactions. The spin degeneracy in zero magnetic field appears to be spontaneously lifted due to the so-called 0.7 anomaly. Further, the g-factor for electrons in the QPC is enhanced, and a zero-bias peak in the conductance points to similarities with transport through a Kondo impurity. We report here how these many-body effects depend on QPC geometry. We find a clear relation between the enhanced g-factor and the subband spacing in our QPCs, and can relate this to the device geometry with electrostatic modeling of the QPC potential. We also measured the zero-field energy splitting related to the 0.7 anomaly, and studied how it evolves into a splitting that is the sum of the Zeeman effect, and a field-independent exchange contribution when applying a magnetic field. While this exchange contribution shows sample-to-sample fluctuations and no clear dependence on QPC geometry, it is for all QPCs correlated with the zero-field splitting of the 0.7 anomaly. This provides evidence that the splitting of the 0.7 anomaly is dominated by this field-independent exchange splitting. Signatures of the Kondo effect also show no regular dependence on QPC geometry, but are possibly correlated with splitting of the 0.7 anomaly.
\end{abstract}

E.J. Koop $(\bowtie)$ - A.I. Lerescu · J. Liu · B.J. van Wees ·

C.H. van der Wal

Physics of Nanodevices Group, Zernike Institute for Advanced

Materials, University of Groningen, Nijenborgh 4,

9747 AG Groningen, The Netherlands

e-mail: e.j.koop@rug.nl

D. Reuter · A.D. Wieck

Angewandte Festkörperphysik, Ruhr-Universität Bochum,

44780 Bochum, Germany
Keywords Quantum point contact · 0.7 anomaly · Many-body electron effects $\cdot$ Nanodevices

\section{Introduction}

A quantum point contact (QPC) is a short channel that carries ballistic one-dimensional electron transport between two reservoirs. Its conductance as a function of channel width is quantized $[1,2]$ and shows plateaus at integer multiples of $2 e^{2} / h$, where $e$ is the electron charge and $h$ Planck's constant. This quantization of the conductance can be understood with a noninteracting electron picture. However, there are several features in the conductance that result from many-body interaction effects between electrons. The effective electron g-factor is enhanced and almost all semiconductor QPCs show an additional plateau at $\sim 0.7\left(2 e^{2} / h\right)$, the so-called 0.7 anomaly. Further, electron transport through QPCs tuned to conditions where the 0.7 anomaly appears has similarities with transport through a Kondo impurity. These many-body effects are not yet fully understood, and in particular understanding the 0.7 anomaly has been the topic of on-going research for more than a decade now [3, 4]. A consistent picture of these effects is of interest for spintronics and quantum information proposals where QPCs are a key element, and QPCs are now also a key model system for studies of many-body physics in nanodevices.

Several models have been proposed that relate the 0.7 anomaly to a spontaneous spin splitting in zero magnetic field [5-11], since the 0.7 plateau evolves continuously into the spin-resolved plateau at $0.5\left(2 e^{2} / h\right)$ when an inplane magnetic field is applied. A recent theory paper [12] presented spin-density functional calculations of realistic QPC geometries that show that a localized state can exist near pinch-off in a QPC, providing a theoretical back- 
ground for the Kondo-like physics that was found experimentally [13]. Other studies have proposed electron-phonon scattering [14], Wigner crystal formation [15], or a dynamical Coulomb blockade effect [16] as the microscopic origin of the 0.7 anomaly. Graham et al. reported evidence that many-body effects also play a role in magnetic fields at crossings between Zeeman levels of different subbands [17], and at crossings of spin-split subbands with reservoir levels [18].

We report here how these many-body effects in QPCs depend on the QPC geometry. We study the energy spacing between the one-dimensional subbands and spin-splittings within one-dimensional subbands, both in zero field and high magnetic fields. While this type of data from individual devices has been reported before $[6,19]$, we report here data from a set of 12 QPCs with identical material parameters. Our measurements show a clear correlation between the subband spacing $\hbar \omega_{12}$ and the enhancement of the effective g-factor $\left|g^{*}\right|$. Both also depend in a regular manner on the geometry of the QPC, and we can understand this behavior using electrostatic modeling of the QPC potential.

The appearance of the 0.7 anomaly and signatures of the Kondo effect do not show a regular dependence on QPC geometry. Intriguingly, however, we find that in high magnetic fields there is a field-independent exchange contribution to the spin-splitting for the lowest one-dimensional subband, in addition to the regular Zeeman splitting, and this exchange contribution is clearly correlated with the zero-field splitting of the 0.7 anomaly. This new observation provides evidence that the splitting of the 0.7 anomaly is dominated by this field-independent exchange splitting. The Kondo effect appears as a zero-bias peak in the differential conductance $G$, and the width of this peak is set by the Kondo temperature $T_{\mathrm{K}}$, an energy scale that represents the strength of the Kondo effect. Our measurements of $T_{\mathrm{K}}$ suggest a correlation between $T_{\mathrm{K}}$ and the splitting of the 0.7 anomaly.

This paper is organized as follows. Section 2 presents information about sample fabrication and measurement techniques. In Sect. 3, we present measurements of the conductance of our set of QPCs, and we extract the energy splittings between subbands and spin splittings. In Sect. 4, we focus on analyzing the signatures of many-body effects in our QPC data, before ending with concluding remarks in the last section.

\section{Experimental Realization}

Our devices were fabricated using a $\mathrm{GaAs} / \mathrm{Al}_{0.32} \mathrm{Ga}_{0.68} \mathrm{As}$ heterostructure with a $2 \mathrm{DEG}$ at $114 \mathrm{~nm}$ below the surface from modulation doping with $\mathrm{Si}$. At $4.2 \mathrm{~K}$, the mobility of the $2 \mathrm{DEG}$ was $\mu=159 \mathrm{~m}^{2} / \mathrm{Vs}$, and the electron density $n_{s}=(1.5 \pm 0.1) \times 10^{15} \mathrm{~m}^{-2}$. A QPC is formed by applying a negative gate voltage $V_{g}$ to a pair of electrodes on the wafer
Table 1 Dimensions of the measured QPCs. The QPC length $L$ and width $W$ are defined as in Fig. 1c

\begin{tabular}{lllllllll}
\hline Device 1 & & & & & & & & \\
\hline$L(\mathrm{~nm})$ & 100 & 150 & 200 & 250 & 300 & 350 & 400 & 450 \\
$W(\mathrm{~nm})$ & 350 & 350 & 350 & 350 & 350 & 350 & 350 & 350 \\
Device 2 & & & & & & & & \\
\hline$L(\mathrm{~nm})$ & 300 & 300 & 300 & 300 & 300 & 300 & 300 & 300 \\
$W(\mathrm{~nm})$ & 200 & 250 & 300 & 350 & 400 & 450 & 500 & 550 \\
\hline
\end{tabular}

surface. The 2DEG below the electrodes is then fully depleted, and tuning of $V_{g}$ allows for controlling the width of a short one-dimensional transport channel. Our QPCs had different values for the length $L$ and width $W$ for the electrode spacing that defines the device (see Table 1, and Fig. 1b, c). Note that $W$ should not be confused with the actual width of the transport channel that is controlled with $V_{g}$. The depletion gates were defined with standard electron-beam lithography and lift-off techniques, using deposition of $15 \mathrm{~nm}$ of Au with a Ti sticking layer. The reservoirs were connected to macroscopic leads via Ohmic contacts, which were realized by annealing a thin $\mathrm{Au} / \mathrm{Ge} / \mathrm{Ni}$ layer that was deposited on the surface.

All QPCs were fabricated in close proximity of each other on a single central part of the wafer to ensure the same heterostructure properties for all QPCs. The set of 8 QPCs for which we varied $L$ (Device 1 in Table 1) had all QPCs within a range of about $10 \mu \mathrm{m}$. The set of 8 QPCs for which we varied $W$ (Device 2 in Table 1 and Fig. 1b) had an identical layout, and was positioned at $2 \mathrm{~mm}$ from Device 1 . Thus, all semiconductor processing steps (resist spinning, e-beam lithography, metal deposition, etc.) could be kept nominally identical for all 16 QPCs. Electron-microscope inspection of the measured devices (after the measurements) confirmed that the dimensions of all gate electrodes were within $10 \mathrm{~nm}$ of the designed values (see Table 1). In our data, this appears as a very regular dependence of QPC properties (see, for example, the discussion of the pinch-off voltage $V_{p o}$ and subband spacing $\hbar \omega_{12}$ in the next section) on $L$ and $W$ for QPCs within the sets of Device 1 and 2. At the same time, two devices from two different sets with nominally identical values of $L$ and $W$ (labeled (1) and (2) in Figs. 3 and 4) show slightly different QPC properties (in particular for the subband spacing $\left.\hbar \omega_{12}\right)$. This is not fully understood.

Measurements were performed in a dilution refrigerator with the sample at temperatures from $\sim 5 \mathrm{mK}$ to $4.2 \mathrm{~K}$. For all our data, the temperature dependence saturated when cooling below $\sim 200 \mathrm{mK}$. We, therefore, assume for this report that this is the lowest effective electron temperature that could be achieved. For measuring the differential conductance $G$, we used standard lock-in techniques at $380 \mathrm{~Hz}$, with an ac voltage bias $V_{a c}=10 \mu \mathrm{V}$. Only the $V_{-}$contact 

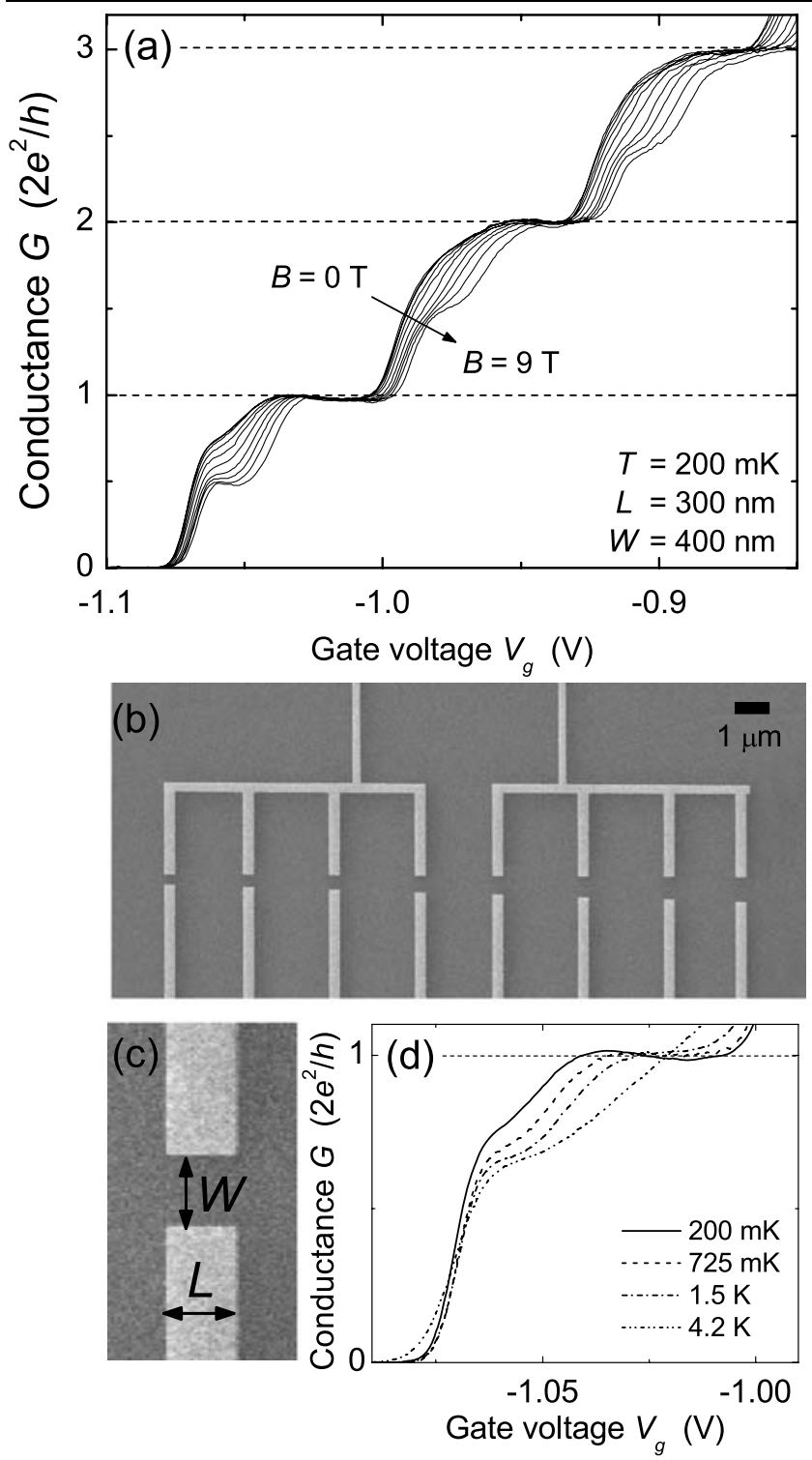

Fig. 1 a The differential conductance $G$ as a function of gate voltage $V_{g}$ at $200 \mathrm{mK}$, for a QPC with $L=300 \mathrm{~nm}$ and $W=400 \mathrm{~nm}$. The in-plane magnetic field is increased from $B=0 \mathrm{~T}$ to $B=9 \mathrm{~T}$. The first three spin-degenerate plateaus at integer multiples of $2 e^{2} / h$ for $B=0 \mathrm{~T}$ split into six spin-resolved plateaus integer multiples of $e^{2} / h$ for $B=9$ T. b Micrograph of a device containing 8 QPCs. From left to right the width $W$ is increased, where $W$ is defined as the spacing between the gate electrodes as shown in (c). $L$ is the length of the channel. Table 1 contains all values for $L$ and $W$ of the measured devices. d Differential conductance $G$ as a function of gate voltage $V_{g}$ at zero field for different temperatures. The 0.7 anomaly becomes more pronounced with increasing temperature

was connected to the grounded shielding of our setup, and all gate voltages were applied with respect to this ground. The in-plane magnetic field was applied perpendicular to the current direction, and the current in the QPCs was along the [110] crystal orientation. Alignment of the sample with the magnetic field was within $1^{\circ}$, as determined from Hall voltage measurements on the 2DEG. We have data from 12 dif- ferent QPCs from the set of 16 that we cooled down. From these QPCs, four could not be measured. For two, this was due to the presence of strong telegraph noise in conductance signals. Two other QPCs did not show clear conductance plateaus.

For analyzing QPC conductance values, we subtracted a magnetic field and temperature dependent series resistance (from the wiring and filters, Ohmic contacts and 2DEG) from the transport data that was obtained with a voltage-bias approach. The criterion here was to make the observed conductance plateaus coincide with integer multiples of $2 e^{2} / h$ or $e^{2} / h$.

\section{Spin Splitting and Energy Splitting Between QPC Subbands}

\subsection{QPC Conductance and Energy Splittings}

Figure 1a presents the differential conductance $G$ of a QPC as a function of $V_{g}$, with the source-drain voltage $V_{s d} \approx 0$. Increasing $V_{g}$ from pinch-off $(G=0)$ lowers and widens the saddle-point-like potential that defines the short transport channel. Consequently, an increasing number of onedimensional subbands gets energies below the Fermi level. In zero magnetic field, this results in a step of $2 e^{2} / h$ in the conductance each time an additional subband starts to contribute to transport. We label these spin-degenerate subbands with a number $N$, starting with $N=1$ for the lowest subband. With a high in-plane magnetic field $B$, the spin degeneracy within each subband $N=1,2,3, \ldots$ is lifted, and the conductance increases now in steps of $e^{2} / h$.

We use this type of data to determine the energy splitting $\Delta E$ between spin-up and spin-down levels within the subbands $N=1,2,3$, and the spacing $\hbar \omega_{12}$ between the $N=1$ and $N=2$ subband (a measure for the degree of transverse confinement in the channel). The onset of transport through a next (spin-polarized) subband appears as a peak in transconductance $\left(\mathrm{d} G / \mathrm{d} V_{g}\right)$ traces as in Fig. 2a, c, e, which we derive from traces as in Fig. 1a. We assume that each subband contributes in a parallel manner to the QPC conductance, and the transconductance curves can then be analyzed as a superposition of peaks, with one (two) peak(s) per (spin-split) subband. We then determine the peak spacings $\Delta V_{g}$ along the $V_{g}$ axis by fitting one or two peaks per subband on the transconductance traces (using least squares fitting with a Gaussian peak shape). The specific shape of a step between the quantized conductance plateaus depends on the shape of the saddle-point-like potential that defines the QPC [20], and can result in asymmetric transconductance peaks. We checked that this is not a significant effect for our analysis.

Subsequently, transconductance data (not shown) from nonlinear transport measurements is used for converting 

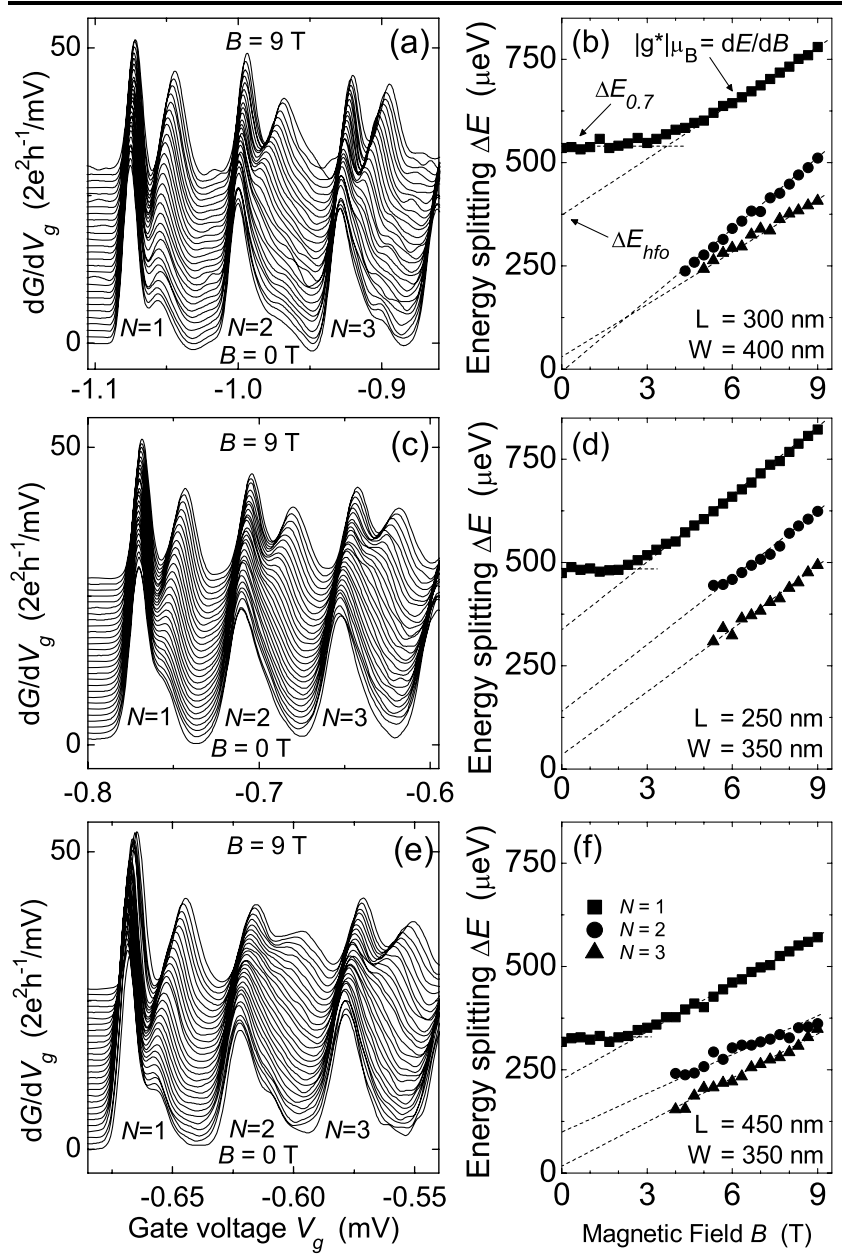

Fig. 2 a Transconductance $\mathrm{d} G / \mathrm{d} V_{g}$ traces (offset vertically) obtained from the data in Fig. 1a (from the QPC with $L=300 \mathrm{~nm}$ and $W=400 \mathrm{~nm}$ ). The 0.7 anomaly appears as a splitting of the transconductance peak for the $N=1$ subband at $B=0 \mathrm{~T}$. b Energy splittings $\Delta E$ obtained from the transconductance traces in (a), as a function of magnetic field. The traces present $\Delta E$ for the subbands $N=1,2,3$, see the legend in (f). These $\Delta E$ traces are characterized (results presented in Fig. 4) with two or three parameters for each subband $N=1,2,3$ : An effective g-factor $\left|g^{*}\right|$, the offset from a linear Zeeman effect in high fields, characterized by the high-field offset $\Delta E_{\text {hfo }}$, and for $N=1$ at low fields the energy splitting of the 0.7 anomaly, $\Delta E_{0.7}$. See text for details. c-f Transconductance traces $\mathrm{d} G / \mathrm{d} V_{g}$ and energy splittings $\Delta E$ as in (a), (b) obtained for a QPC with $L=250 \mathrm{~nm}$ and $W=350 \mathrm{~nm}$ in (c), (d) and $L=450 \mathrm{~nm}$ and $W=350 \mathrm{~nm}$ in (e), (f). All data from measurements at $200 \mathrm{mK}$

$\Delta V_{g}$ values into energy splittings [21]. Here, the onsets of conductance plateaus appear as diamond shaped patterns in the $V_{s d}-V_{g}$ plane. The width of these diamonds along the $V_{s d}$ axis defines the subband spacing, and we use this to determine the spacing $\hbar \omega_{12}$ between the $N=1$ and $N=2$ subband. The slopes of the diamonds can be used to convert a gate-voltage scale into energy scale [21]. In this analysis of $\hbar \omega_{12}$ and conversion of $\Delta V_{g}$ into spin splittings $\Delta E$, we observed a weak dependence on magnetic field and temperature, and took this in account.
The 0.7 anomaly is clearly visible in the data set presented in Fig. 1. The conductance trace for zero field in Fig. 1a shows besides pronounced steps of $2 e^{2} / h$ an additional shoulder at $\sim 0.7\left(2 e^{2} / h\right)$, which becomes more pronounced at higher temperatures (Fig. 1d). With increasing magnetic field, the 0.7 anomaly evolves into the first spinresolved conductance plateau at $e^{2} / h$. In Fig. 2a, c, e, the 0.7 anomaly appears as a zero-field splitting in the transconductance peak for $N=1$, which evolves into two spin-split peaks in high fields. In earlier work, this observation was the basis for assuming that the 0.7 anomaly results from a spontaneous removal of spin degeneracy in zero field $[5,6]$. For our analysis here, we assume that the 0.7 anomaly is indeed related to such a spontaneous spin splitting for the first subband. In high fields, all 12 QPCs showed also for $N=2$ and higher a pronounced spin splitting into two transconductance peaks, but these subbands did not clearly show a zero-field splitting.

We studied how the spin splittings $\Delta E$ for $N=1,2,3$ increase with magnetic field from $B=0 \mathrm{~T}$ up to $9 \mathrm{~T}$ (Fig. 2b, d, f). We first concentrate on data for $N=1$. At zero field, $\Delta E$ shows the splitting associated with the 0.7 anomaly, that we label $\Delta E_{0.7}$. It is observed in all our QPCs with a typical value of $0.5 \mathrm{meV}$. At high fields, $\Delta E$ has a linear slope similar to the Zeeman effect. However, linear extrapolation of this slope down to $B=0$ shows that there is a large positive offset (unlike the usual Zeeman effect). We characterize the slope with an effective g-factor $\left|g^{*}\right|=\frac{1}{\mu_{B}} \frac{\mathrm{d} \Delta E}{\mathrm{~d} B}$ (note that one should be careful to interpret $\left|g^{*}\right|$ as an absolute indication for the g-factor of electrons in a QPC, since different methods for extracting a g-factor can give different results $[13,19])$. The high-field offset from a linear Zeeman effect is characterized with a parameter $\Delta E_{h f o}$. Qualitatively, this type of data for $\Delta E$ looks similar for all 12 QPCs (Fig. 2b, $\mathrm{d}, \mathrm{f})$, and we use a suitable fitting procedure to characterize the traces for $N=1$ with the parameters $\Delta E_{0.7}, \Delta E_{h f o}$ and $\left|g^{*}\right|$. Notably, two-parameter fitting using spin- $\frac{1}{2}$ energy eigenvalues with $\Delta E=\sqrt{\left(\Delta E_{0.7}\right)^{2}+\left(\left|g^{*}\right| \mu_{B} B\right)^{2}}$ does not yield good fits. For the traces as in Fig. 2b, d, f for $N=2,3$, we cannot resolve a spin splitting at low fields, only the parameters $\Delta E_{h f o}$ and $\left|g^{*}\right|$ can be derived. Further analysis of these results for $\Delta E_{0.7}, \Delta E_{h f o}$ and $\left|g^{*}\right|$ is presented in Sect. 4 on many-body effects.

\subsection{Electrostatics and Subband Splitting}

Figure $3 \mathrm{a}, \mathrm{b}$ presents how the pinch-off voltage $V_{p o}$ (the value of $V_{g}$ where the $G$ starts to increase from zero) depends on QPC geometry. Figure 3c, d presents this for the subband spacing $\hbar \omega_{12}$, which provides a parameter for the strength of the transverse confinement in the QPC, and is possibly of importance for several of the many-body effects in QPCs. The observed dependence of $V_{p o}$ on the QPC 

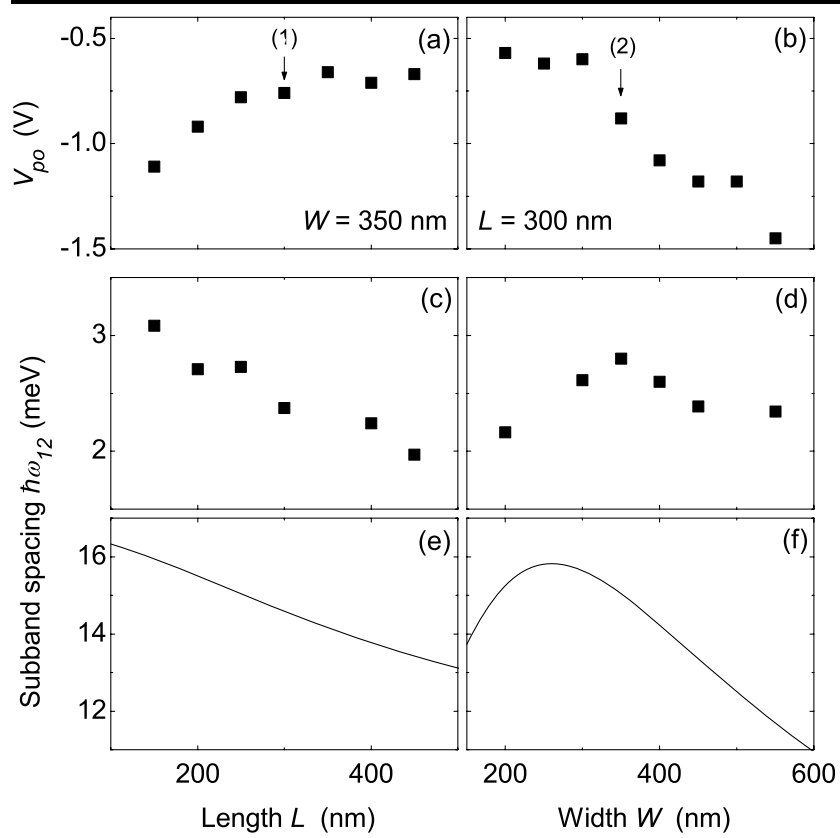

Fig. 3 a, b The pinch-off voltage $V_{p o}$ as a function of QPC length $L$ (with fixed width $W=350 \mathrm{~nm}$ ), and as a function QPC width $W$ (with fixed length $L=300 \mathrm{~nm}$ ). Data points labeled with (1) and (2) are from two different devices with nominally identical values for $L$ and $W$ (see text for details). $\mathbf{c}, \mathbf{d}$ The measured subband spacing $\hbar \omega_{12}$ as a function of $L$ and $W$. e, f Calculated subband spacing $\hbar \omega_{12}$ as a function of $L$ and $W$, from electrostatic modeling of the QPC potential. The results qualitatively reproduce the trend in the experimental data in $(\mathbf{c}),(\mathbf{d})$

geometry (a more negative $V_{p o}$ for shorter and wider QPCs) agrees with the expected trend. This provides the first of several indications that part of the physics of our set of QPCs depends in a regular manner on $L$ and $W$.

Furthermore, the variation of $\hbar \omega_{12}$ is in good agreement with an electrostatic analysis [22] of the degree of transverse confinement in the saddle-point-like potential of the QPC (presented in Fig. 3e, f). In summary, short and narrow QPCs yield the strongest transverse confinement (Fig. 3d-f). This is valid down to the point where the QPC width $W \lesssim 3 d$ (where $d$ the depth of the 2DEG below the wafer surface), which results in the maximum for $W=350 \mathrm{~nm}$ in Fig. 3d, f.

For this analysis, we calculate the confining electrostatic potential due to the depletion gates in the plane of the 2DEG. An important ingredient of the calculation is the threshold voltage $V_{t}$, which is the (negative) voltage that must be applied to a gate to reduce the electron density underneath it to zero,

$V_{t}=-\frac{e n_{2 D} d}{\epsilon_{r} \epsilon_{0}}$,

where $n_{2 D}$ is the 2DEG electron density (without gates) and $d$ the depth of the 2DEG below the surface. We use the dielectric constant for GaAs $\epsilon=\epsilon_{r} \epsilon_{0}=12.9$.

The subband spacing can be calculated from the transverse curvature of the saddle-point-like potential. However, this curvature changes with the applied gate voltage. Therefore, we calculate the curvature for all QPCs when the QPC is just pinched-off ( $G=0)$, when the potential in the middle of the transport channel is equal to $V_{t}$. For these calculations, we used the dimensions $L, W$ and $d$ of the measured devices. Qualitatively, the trends in $\hbar \omega_{12}$ as a function of $L$ and $W$ are reproduced, but the calculated values for $\hbar \omega_{12}$ are significantly larger than the experimentally obtained values. From earlier work [22], it is known that it is hard to get quantitative agreement from this type of calculations. Furthermore, the maximum in $\hbar \omega_{12}$ versus $W$ is found for a smaller value for $W$ than we have observed experimentally, approximately when $W \sim 2 d$.

The origin of differences between our simple calculations and experimental results is well understood. The treatment of the exposed surfaces between the depletion gates is an important aspect of the calculations. A different choice for the boundary condition of the exposed surface may result in a noticeable difference in the confining potential $[22,23]$. We used a so-called pinned-surface approach (because it is a simple analytical approach) where the Fermi level at the surface becomes pinned to the Fermi level in the 2DEG. However, a pinned surface requires charge to move from the 2DEG to the surface when the gate voltage is changed, in order to keep the surface potential constant. This process is strongly suppressed at low temperatures. Alternatively, the surface can be treated as a dielectric boundary, with a fixed charge density (frozen surface approach). Davies et al. [22] have compared the results for pinned and frozen surfaces and found that the maximum in $\hbar \omega_{12}$ shifts from $W \sim 2 d$ to $W \sim 3 d$ when a frozen surface is assumed instead of a pinned surface. This corresponds very well to the experimentally observed value of $350 \mathrm{~nm}$. Furthermore, the model used here is based on the calculation of the electrostatic potential due to the gates alone. Other effects, as the contribution to the potential from donor ions and other electrons in the 2DEG are ignored. Self-consistent calculations [24] have shown that the values of $\hbar \omega_{12}$ decrease rapidly when electrons enter the conduction channel.

\section{Many-Body Effects}

\subsection{Enhancement of the Effective g-Factor}

Figure $4 \mathrm{a}, \mathrm{b}$ presents how the effective g-factor $\left|g^{*}\right|$ for $N=1$ varies with $L$ and $W$ of the QPCs. It is strongly enhanced up to a factor $\sim 3$ with respect to the g-factor for bulk 2DEG material [25] (the temperature dependence of this $\left|g^{*}\right|$ data is shortly discussed in Sect. 4.2). This has been observed before [6] and is attributed to many-body effects. Notably, the values of $\left|g^{*}\right|$ and $\hbar \omega_{12}$ in Fig. 3c, $d$ are clearly correlated. 

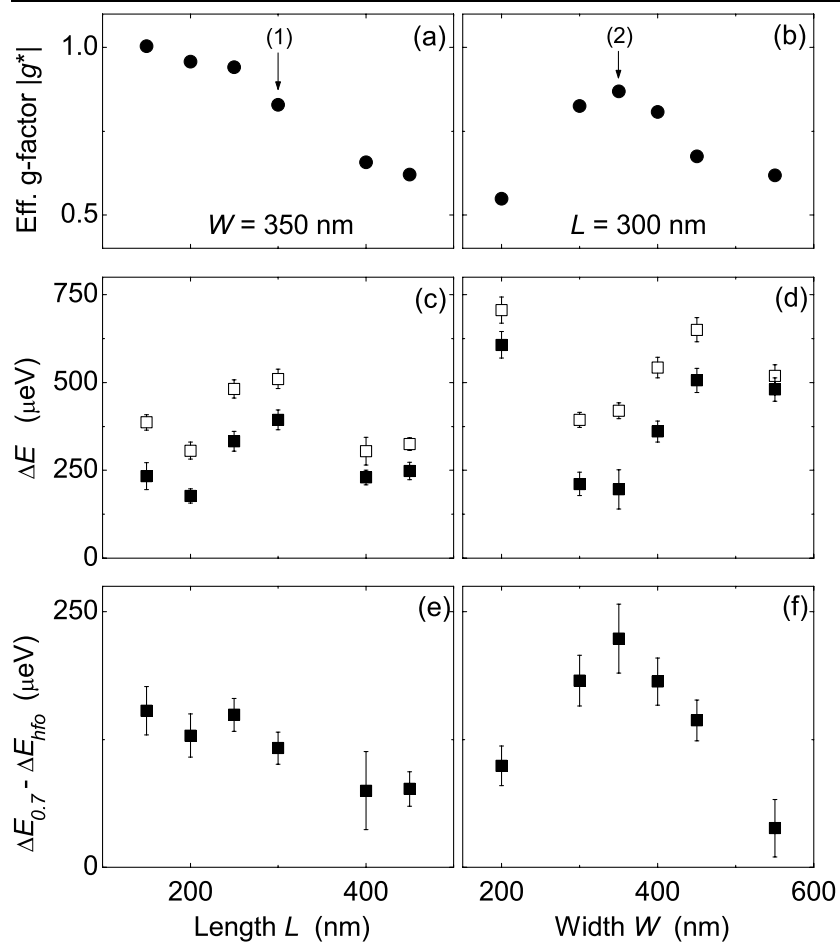

Fig. 4 a, b Effective g-factor $\left|g^{*}\right|$ as a function of QPC length $L$ (with fixed width $W=350 \mathrm{~nm}$ ), and as a function QPC width $W$ (with fixed length $L=300 \mathrm{~nm}$ ). The effective g-factor $\left|g^{*}\right|$ is enhanced as compared to the bulk 2DEG value (up to a factor $\sim 3$ ) and shows a clear dependence on $L$ and $W$, that is correlated with the dependence of the subband spacing $\hbar \omega_{12}$ in Fig. 3c, d. Data points labeled with (1) and (2) are from two different devices with nominally identical values for $L$ and $W$ (see text for details). c, $\mathbf{d}$ The 0.7 energy splitting $\Delta E_{0.7}$ and high-field offset $\Delta E_{\text {hfo }}$ for the $N=1$ subband as a function of $L$ and $W$. The values of $\Delta E_{0.7}$ and $\Delta E_{h f o}$ both vary with $L$ and $W$ in a irregular manner, but there is a strong correlation between $\Delta E_{0.7}$ and $\Delta E_{h f o}$. e, $\mathbf{f}$ The difference between $\Delta E_{0.7}$ and $\Delta E_{h f_{o}}$ as a function of $L$ and $W$. This data again shows a correlation with the dependence of subband spacing $\hbar \omega_{12}$ on $L$ and $W$. All data points are for the $N=1$ subband from results measured at $200 \mathrm{mK}$ (Fig. 3c, d)

The enhancement of the effective g-factor has been explained in terms of exchange interactions (see [26] and references therein). Calculations of the exchange potential in a square (quantum well) confining potential have shown that the effective g-factor decreases when the 1D confining potential weakens and the 2D limit is approached [27]. For a harmonic confining potential, the results of this study predict that $\left|g^{*}\right|$ scales indeed with $\hbar \omega_{12}$. We observed this here for the lowest subband $(N=1)$ in dependence on QPC geometry. Earlier work [6] observed the same trend in a single QPC, using that the transverse confinement decreases with increasing subband index $N$.

\subsection{The 0.7 Anomaly and Exchange}

Figure $2 \mathrm{~b}, \mathrm{~d}$, f show that for all QPCs $\Delta E$ appears in high fields as the sum of the Zeeman effect and the constant contribution $\Delta E_{h f o}$. This suggest that the splittings in high field have, in particular for $N=1$, a significant contribution from a field-independent exchange effect that results from each subband being in a ferromagnetic spin-polarized state. In high fields, such an interpretation is less ambiguous than for zero field (where the possibility of a ferromagnetic ground state for spin-polarized subbands is the topic of debate $[28,29])$ since the Zeeman effect suppresses spin fluctuations. Thus, measuring $\Delta E_{h f_{o}}$ can be used to determine this exchange splitting.

We now further analyze how this parameter, $\Delta E_{\text {hfo }}$ and $\Delta E_{0.7}$, depend on $L$ and $W$. The open squares in Fig. $4 \mathrm{c}, \mathrm{d}$ present this for $\Delta E_{0.7}$. Overall, the dependence here is not very regular, possibly indicating that the exact appearance of the otherwise robust 0.7 anomaly is sensitive to small irregularities in the potential that defines the QPC (only the data in Fig. 4d suggests an anti-correlation with $\left.\hbar \omega_{12}\right)$. The black squares present how $\Delta E_{h f o}$ for $N=1$ varies with $L$ and $W$. Also here the dependence is irregular. Remarkably, however, the irregular variations of $\Delta E_{0.7}$ and $\Delta E_{h f o}$ are clearly correlated throughout our set of 12 QPCs. This means that $\Delta E_{0.7}$, which is derived from data in zero field, is correlated with $\Delta E_{h f o}$, which is derived from data taken at fields in excess of $5 \mathrm{~T}$. Further evidence for the significance of this correlation comes from data from the $N=2$ and $N=3$ subband (see Fig. 2b, d, f). We analyzed the data for $N=2,3$ in the very same way as for $N=1$, and the most important observation is that the $\Delta E_{h f o}$ parameter for $N=2,3$ is much smaller than for $N=1$, and often close to zero. A high $\Delta E_{\text {hfo }}$ value is only observed for $N=1$, just as the 0.7 anomaly itself. Notably, for $N=1, \Delta E_{0.7}$ and $\Delta E_{h f o}$ also have a similar order of magnitude. This analysis points to the conclusion that the spontaneous energy splitting of the 0.7 anomaly is dominated by the same effect that causes the high-field offset $\Delta E_{h f o}$. As we discussed, this is probably an exchange contribution [30]. The error bar that we attribute to these values includes an error from the transconductance peak-fitting, one from the conversion of gate voltage to energy scale, and an error due to scatter in the $\Delta E$ datapoints as a function of $B$.

Figure $4 \mathrm{e}, \mathrm{f}$ presents data for the difference between $\Delta E_{0.7}$ and $\Delta E_{h f o}$. Here, $\Delta E_{0.7}-\Delta E_{h f o}$ shows again a correlation with $\hbar \omega_{12}$. This indicates that the splitting of the 0.7 anomaly has (in addition to the exchange contribution that is also present in high fields) a contribution that scales with $\hbar \omega_{12}$. At this stage, we cannot relate this new observation to earlier experimental or theoretical work. Note that for the error bars in Fig. 4e, f, we first subtracted the values of peak positions in terms of gate voltage, such that the error from gate voltage to energy scale conversion is accounted for only once.

We will now discuss the effect of increasing the temperature on the many-body phenomena in our QPCs. Figure 5a-d shows the conductance $G$ at $V_{s d} \sim 0$ as a function 

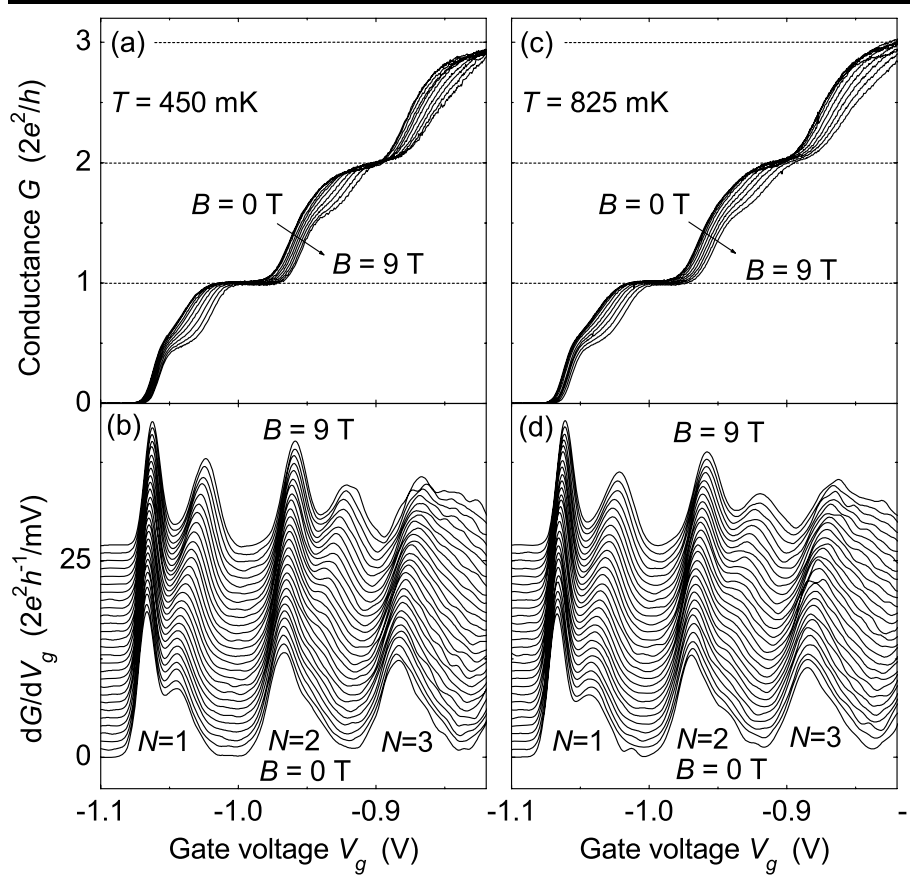

Fig. 5 a Differential conductance $G$ as a function of gate voltage $V_{g}$ at $450 \mathrm{mK}$, for a QPC with $L=300 \mathrm{~nm}$ and $W=400 \mathrm{~nm}$. The in-plane magnetic field is increased from $B=0 \mathrm{~T}$ to $B=9 \mathrm{~T}$. b Transconductance $\mathrm{d} G / \mathrm{d} V_{g}$ traces (offset vertically for clarity) obtained by

of magnetic field for temperatures $T=450 \mathrm{mK}, 825 \mathrm{mK}$, $1.5 \mathrm{~K}$ and $2.8 \mathrm{~K}$ (see also Figs. 1a and 2a for the $200 \mathrm{mK}$ data). As the temperature is increased the spin-degenerate plateaus and the spin-resolved plateaus both become less pronounced due to thermal smearing. In high magnetic fields, the spin-resolved plateaus increase slightly in conductance with increasing temperature. At even higher temperatures, the plateau at $0.7\left(2 e^{2} / h\right)$ is the last remaining feature in the differential conductance. Notably, here the 0.7 anomaly appears to be present over the whole range of magnetic fields. The corresponding transconductance traces $\mathrm{d} G / \mathrm{d} V_{g}$ are plotted in Fig. 5e-h. As a result of the thermal smearing of the conductance plateaus, the peaks in $\mathrm{d} G / \mathrm{d} V_{g}$ become broader and decrease in height. The zero-field splitting in the transconductance peak for $N=1$ has been identified as the 0.7 anomaly. When the temperature is increased, the 0.7 anomaly becomes more pronounced as was shown in the temperature dependence of the differential conductance $G$ presented in Fig. 1d. Consequently, the zero-field splitting in Fig. 5e-h also increases. For $T=825 \mathrm{mK}$ and $1.5 \mathrm{~K}$ (Fig. 5f, g) even a small zero-field splitting of the $N=2$ transconductance peak can be observed, suggesting the appearance of a $1.7\left(2 e^{2} / h\right)$ plateau [6].

Using the temperature dependence of $\Delta E$ data (Fig. 6a), we find that the correlation between $\Delta E_{0.7}$ and $\Delta E_{h f o}$ remains intact at higher temperatures (Fig. 6c). Figure 6b shows that $\left|g^{*}\right|$ has a very different temperature dependence.
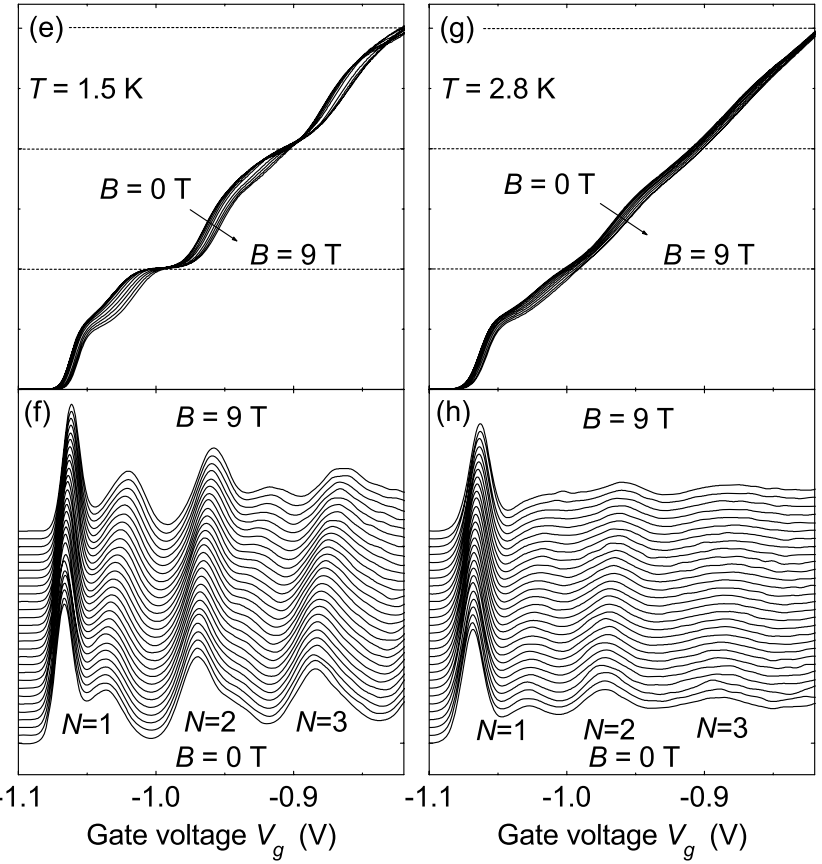

differentiating the data in (a). The conductance $G$ and transconductance $\mathrm{d} G / \mathrm{d} V_{g}$ as in (a), (b) are shown for $T=825 \mathrm{mK}$ in (c), (d), for $T=1.5 \mathrm{~K}$ in $(\mathbf{e}),(\mathbf{f})$ and for $T=2.8 \mathrm{~K}$ in $(\mathbf{g}),(\mathbf{h})$

This indicates that the g-factor enhancement and the 0.7 anomaly arise from different many-body effects.

\subsection{Kondo Signatures}

The appearance of the 0.7 anomaly has been related to a peak in the differential conductance as a function of sourcedrain voltage around zero bias, for $G$ values around $e^{2} / h$. Earlier work [13] showed that this zero-bias anomaly (ZBA), and its temperature and magnetic field dependence, have a very striking similarity with electron transport through a Kondo impurity that can studied with quantum dots [31, 32]. For quantum dots, the Kondo effect is a manybody interaction of the localized electron(s) inside the dot with the delocalized electrons in the leads connected to the dot [13, 31-33]. Together these electrons form a spin-singlet state, effectively screening the local spin on the dot. In contrast to a quantum dot, where there is a clear localized state, a QPC is an open system where the formation of a bound state is much less obvious. A recent theoretical result [12] has shown that a self-consistent many-body state can indeed form inside a QPC, and that this can result in Kondo-like physics.

In this section, we present the measurements of this ZBA in our set of QPCs. Most of our QPCs showed a clear ZBA in nonlinear conductance measurements. The temperature and magnetic field dependence of this data (Fig. 7a-d) is consis- 

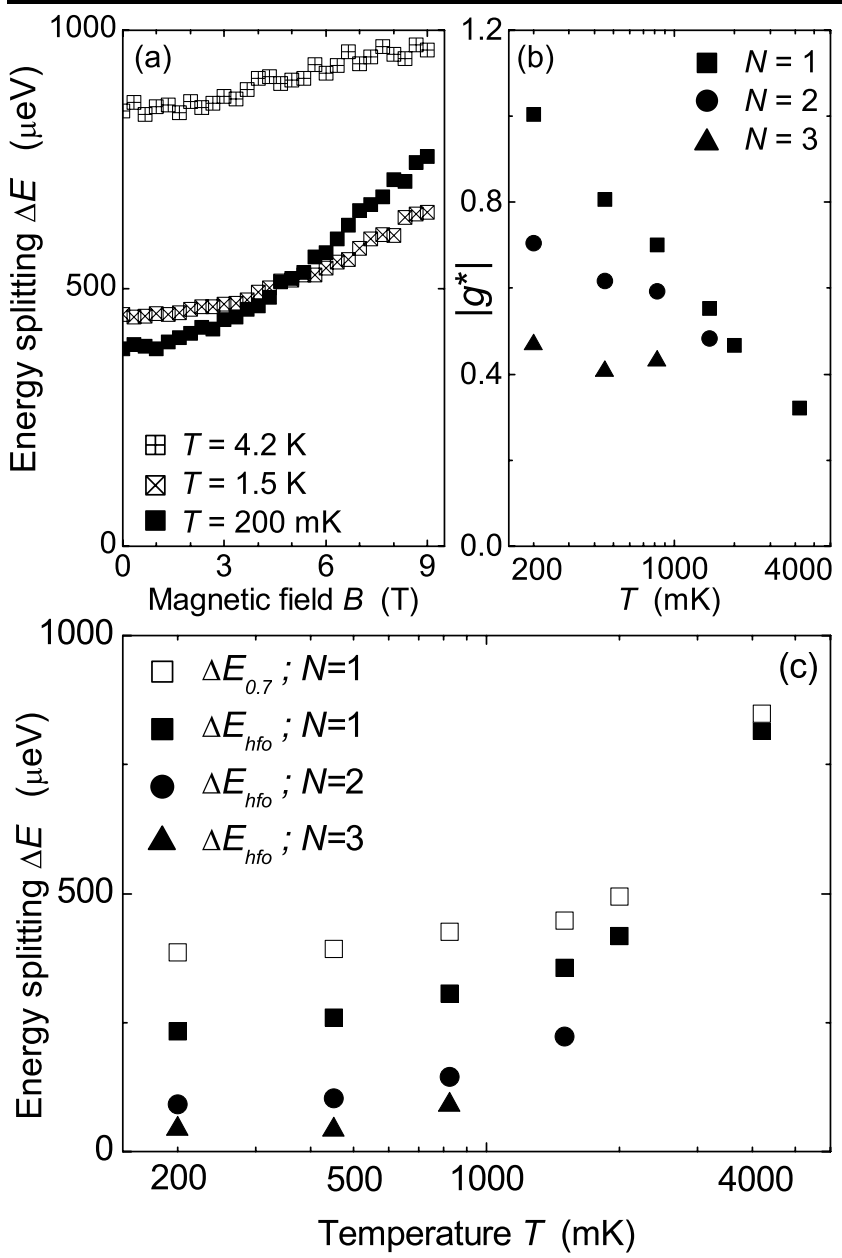

Fig. 6 a Energy splitting for $N=1$ as a function of magnetic field for different temperatures $T$, for a QPC with $L=150 \mathrm{~nm}$ and $W=350 \mathrm{~nm}$. b Effective g-factor $\left|g^{*}\right|$ as a function of temperature for the same QPC. The g-factor enhancement is strongest for the $N=1$ subband at the lowest temperature. For the $N=2$ and $N=3$ subband the g-factor is also enhanced at low temperatures. As the temperature is increased the $\mathrm{g}$-factor enhancement is weaker for all subbands. $\mathbf{c}$ The 0.7 energy splitting $\Delta E_{0.7}$ and high-field offset $\Delta E_{h f o}$ as a function of temperature. The value for $\Delta E_{h f o}$ is highest for the $N=1$ subband and decreases to zero with increasing subband number. As the temperature is increased, the $\Delta E_{0.7}$ value as well as the $\Delta E_{h f o}$ values for $N=1,2,3$ strongly increase. The correlation between $\Delta E_{0.7}$ and $\Delta E_{\text {hfo }}$ remains present upon increasing the temperature

tent with the earlier reports [13] that relate the 0.7 anomaly to transport through a Kondo impurity.

The relevant energy scale for Kondo physics is the Kondo temperature $T_{\mathrm{K}}$. Below this temperature, the magnetic impurity giving rise to Kondo physics is completely screened by the formation of a spin-singlet state and at zero-bias the differential conductance $G \cong 2 e^{2} / h$. The Kondo temperature determines the width of the zero-bias peak. We observe that the peak width and height $\delta G$ of the ZBA are not constant over the whole range $0<\mathrm{G}<2 \mathrm{e}^{2} / h$ (see also Figs. 6, 7, 8, $9,10,11$ and 12 in [19]). We choose to fit these parameters at $G \sim 0.3$, where the peak height has a relative maximum. The
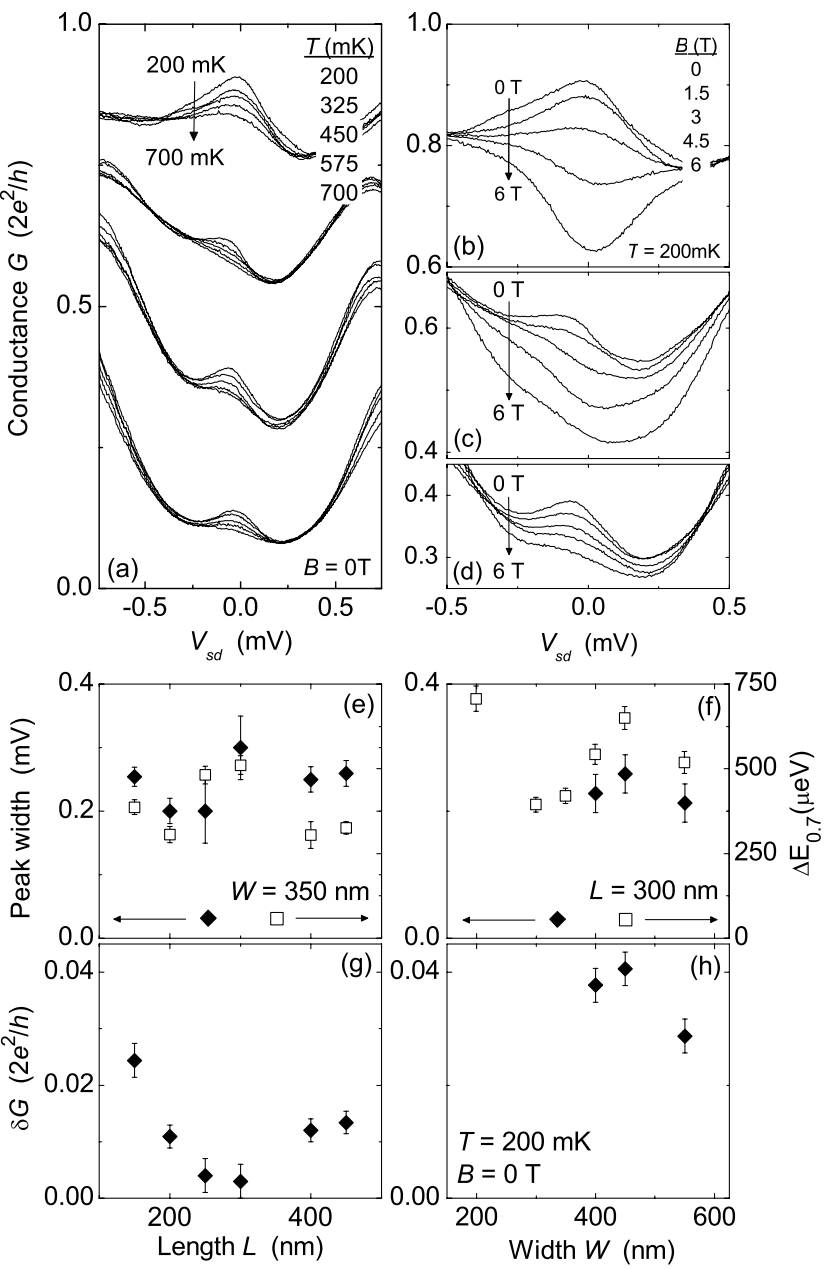

Fig. 7 a Temperature dependence of the zero-bias anomaly (ZBA) for 4 fixed values of the gate voltage. The peak becomes more pronounced as temperature is lowered from 700 to $200 \mathrm{mK}$. b-d Magnetic field dependence of the ZBA for $G \sim 0.8(\mathbf{b}), G \sim 0.5$ (c) and $G \sim 0.3\left(2 e^{2} / h\right)(\mathbf{d})$. The ZBA should split by upon application of an in-plane magnetic field $[13,32]$. The peak in (b) does not split but collapses with $B$ because $T_{K}<2 g^{*} \mu_{B} B$ in this regime [13]. The splitting in (c) and (d) is not very prominent, possibly due to our electron temperature of $\sim 200 \mathrm{mK}$. e, f The width of the ZBA at $G \sim 0.3\left(2 e^{2} / h\right)$ (left axis) as a function of QPC length $L$ and width $W$. The peaks are fitted in zero magnetic field at $T=200 \mathrm{mK}$. The right axis shows $\Delta E_{0.7}$ (data from Fig. $4 \mathrm{~g}, \mathrm{~h}$ ). $\mathbf{g}, \mathbf{h}$ Height of the ZBA also at $G \sim 0.3\left(2 e^{2} / h\right)$ versus $L$ and $W$ for the same conditions as in (e), (f)

peak width and height are determined by fitting the nonlinear conductance traces with a Gaussian shaped peak added to a parabola.

Figure 7e, f shows the peak width as a function of $L$ and $W$ (during our measurement run, one gate of Device 2 broke during an electronic malfunction, and we can only present data from 3 QPCs in the set with different values of $W$ ). The width of the ZBA does not show a clear dependence on $L$ and $W$, and has a value of about $2 \mathrm{mV}$ for all QPCs. For completeness, we also report the peak height $\delta G$ in Fig. $7 \mathrm{~g}, \mathrm{~h}$ as a function of $L$ and $W$. We observe that for 
a single QPC $\delta G$ varies with $V_{g}$, but the values in Fig. $7 \mathrm{~g}$, h do give for each QPC a good representation of the typical value of $\delta G$ throughout the $V_{g}$ interval where the ZBA is observed. As a function of $L$ and $W$, we observe here a stronger scatter in the values than for the peak width, but also here, there is no clear relation with the QPC geometry. To conclude this section, we consider a correlation between the signatures of the Kondo effect and the values of $\Delta E_{0.7}$ (Fig. 7e, f). The irregular variation of the ZBA peak width with $L$ and $W$ suggests indeed a correlation with $\Delta E_{0.7}$, but here the evidence is very weak given the size of the error bar that we attribute to these values.

\section{Discussion and Conclusions}

We have studied many-body interaction effects in quantum point contacts. Our main point of interest was the dependence of these many-body electron interactions on the geometry of the QPC. We found a clear relation between the subband spacing and the enhancement of the effective electron g-factor. These parameters depend on geometry in a regular manner that we can understand from electrostatic modeling of the QPC potential. The many-body electron physics that causes the spontaneous energy splitting of the 0.7 anomaly does not show a clear dependence on QPC geometry, but we do find a clear correlation with a fieldindependent exchange effect that contributes to spin splittings in high magnetic fields. This suggests that the splitting of the 0.7 anomaly is dominated by this exchange contribution. We also measured a zero-bias anomaly in the nonlinear conductance of our QPCs that has been interpreted as a signature of the Kondo effect. Here, there is also no clear dependence on QPC geometry, but our data suggests that it is worthwhile to further study its correlation with the splitting of the 0.7 anomaly. These results are important for theory work that aims at developing a consistent picture of manybody effects in QPCs, and its consequences for transport of spin-polarized electrons and spin coherence in nanodevices. Our analysis of experimental data is very phenomenological, presenting parameters and correlations for which it is difficult to draw conclusions about the underlying physics. At the same time, part of state-of-the-art theory work now relies on numerical simulations of realistic QPC geometries (using spin-density-functional theory [12, 29] or other numerical approaches [28]) from which it is hard to derive analytical expression for the underlying physics. However, the validity of this numerical modeling can be easily tested for its consistency with the parameters and correlations that we reported here.

Acknowledgements We thank B.H.J. Wolfs, R.N. Schouten, S.F. Fischer, C.W.J. Beenakker and L.S. Levitov for help and useful discussions, and the Dutch Foundation for Fundamental Research on Matter (FOM), the Netherlands Organization for Scientific Research (NWO), and the German BMBF (in the framework of the nanoQUIT-program) for financial support.

\section{References}

1. van Wees, B.J., van Houten, H., Beenakker, C.W.J., Williamson, J.G., Kouwenhoven, L.P., van der Marel, D., Foxon, C.T.: Phys. Rev. Lett. 60, 848 (1988)

2. Wharam, D.A., Pepper, M., Ahmed, H., Frost, J.E.F., Hasko, D.G., Peacock, D.C., Ritchie, D.A., Jones, G.A.C.: J. Phys. C 21, L209 (1988)

3. Fitzgerald, R.: Phys. Today. 55, 21 (2002)

4. Ensslin, K.: Nat. Phys. 2, 587 (2006)

5. Thomas, K.J., Nicholls, J.T., Simmons, M.Y., Pepper, M., Mace, D.R., Ritchie, D.A.: Phys. Rev. Lett. 77, 135 (1996)

6. Thomas, K.J., Nicholls, J.T., Appleyard, N.J., Simmons, M.Y., Pepper, M., Mace, D.R., Tribe, W.R., Ritchie, D.A.: Phys. Rev. B 58, 4846 (1998)

7. Kristensen, A., Bruus, H., Hansen, A.E., Jensen, J.B., Lindelof, P.E., Marckmann, C.J., Nygård, J., Sørensen, C.B., Beuscher, F., Forchel, A., Michel, M.: Phys. Rev. B 62, 10950 (2000)

8. Meir, Y., Hirose, K., Wingreen, N.S.: Phys. Rev. Lett. 89, 196802 (2002)

9. Starikov, A.A., Yakimenko, I.I., Berggren, K.-F.: Phys. Rev. B 67, 235319 (2003)

10. Reilly, D.J.: Phys. Rev. B 72, 033309 (2005)

11. DiCarlo, L., Zhang, Y., McClure, D.T., Reilly, D.J., Marcus, C.M., Pfeiffer, L.N., West, K.W.: Phys. Rev. Lett. 97, 036810 (2006)

12. Rejec, T., Meir, Y.: Nature 442, 900 (2006)

13. Cronenwett, S.M., Lynch, H.J., Goldhaber-Gordon, D., Kouwenhoven, L.P., Marcus, C.M., Hirose, K., Wingreen, N.S., Umansky, V.: Phys. Rev. Lett. 88, 226805 (2002)

14. Seelig, G., Matveev, K.A.: Phys. Rev. Lett. 90, 176804 (2003)

15. Matveev, K.A.: Phys. Rev. Lett. 92, 106801 (2004)

16. Bulka, B.R., Kostyrko, T., Tolea, M., Dinu, I.V.: J. Phys.: Condens. Matter 19, 255211 (2007)

17. Graham, A.C., Thomas, K.J., Pepper, M., Cooper, N.R., Simmons, M.Y., Ritchie, D.A.: Phys. Rev. Lett. 91, 136404 (2003)

18. Graham, A.C., Pepper, M., Simmons, M.Y., Ritchie, D.A.: Phys. Rev. B 72, 193305 (2005)

19. Cronenwett, S.M.: Ph.D. thesis, Stanford University (2001)

20. Büttiker, M.: Phys. Rev. B 41, 7906 (1990)

21. Patel, N.K., Nicholls, J.T., Martn-Moreno, L., Pepper, M., Frost, J.E.F, Ritchie, D.A., Jones, G.A.C.: Phys. Rev. B 44, 10973 (1991)

22. Davies, J.H., Larkin, I.A.: J. Appl. Phys. 77, 4504 (1995)

23. Chen, M., Porod, W., Kirkner, D.J.: J. Appl. Phys. 75, 2545 (1993)

24. Laux, S.E., Frank, D.J., Stern, F.: Surf. Sci. 196, 101 (1988)

25. Hannak, R.M., Oestreich, M., Heberle, A.P., Rühle, W.W., Köhler, K.: Solid State Commun. 93, 319 (1995)

26. Pallecchi, I., Heyn, C., Lohse, J., Kramer, B., Hansen, W.: Phys. Rev. B 65, 125303 (2002)

27. Majumdar, A.: J. Appl. Phys. 83, 297 (1998)

28. Klironomos, A.D., Meyer, J.S., Matveev, K.A.: Europhys. Lett. 74, 679 (2006)

29. Jaksch, P., Yakimenko, I., Berggren, K.-F.: Phys. Rev. B $\mathbf{7 4 2}$, 235320 (2006)

30. Koop, E.J., Lerescu, A.I., Liu, J., van Wees, B.J., Reuter, D., Wieck, A.D., van der Wal, C.H.: arXiv:0706.0792v1 (2007); [cond-mat.mes-hall]

31. Goldhaber-Gordon, D., Shtrikman, H., Mahalu, D., AbuschMagder, D., Meirav, U., Kastner, M.A.: Nature 391, 156 (1998)

32. Cronenwett, S.M., Oosterkamp, T.H., Kouwenhoven, L.P.: Science 281, 540 (1998)

33. Kouwenhoven, L.P., Glazman, L.I.: Phys. World 14, 33 (2001) 